\title{
A Low Complexity Subspace-Based DOA Estimation Algorithm with Uniform Linear Array Correlation Matrix Subsampling
}

\author{
Do-Sik Yoo \\ School of Electronic and Electrical Engineering, Hongik University, Mapo-gu, Wausan-ro 94, Seoul 04066, Republic of Korea \\ Correspondence should be addressed to Do-Sik Yoo; yoodosik@hongik.ac.kr
}

Received 9 July 2015; Revised 8 November 2015; Accepted 12 November 2015

Academic Editor: Ding-Bing Lin

Copyright (C) 2015 Do-Sik Yoo. This is an open access article distributed under the Creative Commons Attribution License, which permits unrestricted use, distribution, and reproduction in any medium, provided the original work is properly cited.

\begin{abstract}
We propose a low complexity subspace-based direction-of-arrival (DOA) estimation algorithm employing a direct signal space construction method (DSPCM) by subsampling the autocorrelation matrix of a uniform linear array (ULA). Three major contributions of this paper are as follows. First of all, we introduce the method of autocorrelation matrix subsampling which enables us to employ a low complexity algorithm based on a ULA without computationally complex eigenvalue decomposition or singularvalue decomposition. Secondly, we introduce a signal vector separation method to improve the distinguishability among signal vectors, which can greatly improve the performance, particularly, in low signal-to-noise ratio (SNR) regime. Thirdly, we provide a root finding (RF) method in addition to a spectral search (SS) method as the angle finding scheme. Through simulations, we illustrate that the performance of the proposed scheme is reasonably close to computationally much more expensive MUSIC(MUltiple SIgnal Classification-) based algorithms. Finally, we illustrate that the computational complexity of the proposed scheme is reduced, in comparison with those of MUSIC-based schemes, by a factor of $\mathcal{O}\left(N^{2} / K\right)$, where $K$ is the number of sources and $N$ is the number of antenna elements.
\end{abstract}

\section{Introduction}

Subspace-based spectral estimation and direction-of-arrival (DOA) estimation schemes have been widely studied during the last several decades [1-3]. Right after the introduction of Pisarenko and MUSIC (MUltiple SIgnal Classification) algorithms, various alternative schemes such as minNorm method [4], ESPRIT (Estimation of Signal Parameter via Rotational Invariance Technique) [5], and root-MUSIC method [6] were developed during the first decade. In more recent years, such early ideas and concepts have been extended to deal with various issues such as nonlinear or nonuniform array shapes [7-9], coherent sources [10], and 2dimensional (2D) angle estimation [11]. Among such issues drawing continued research interest is the computational complexity, which is particularly important for arrays with large number of antenna elements.

One of the main reasons for large computational complexity with existing subspace-based algorithms is the computational burden to construct the subspace, which usually involves the process of eigendecomposition (ED) or singular-value decomposition (SVD). To reduce the computational burden that arises due to ED or SVD, various algorithms that do not require ED or SVD have been proposed. Among the first such examples was the method proposed in [12] which computes the projection matrix by using a submatrix of the correlation matrix. Later, in [13], an improved algorithm called orthogonal propagator method has been proposed that achieves better performance particularly at medium and high signal-to-noise ratio (SNR). Recently, a 2-dimensional (2D) DOA estimation algorithm based on cross-correlation matrix was proposed in [14] that similarly computes the required projection operators without using ED or SVD. However, even though these methods do not require ED or SVD process, they are still computationally burdensome particularly for an array with large antenna elements.

More recently, Xi and Liping proposed, in [15], a very impressive 2D DOA estimation algorithm with L-shaped array. Most of all, it is truly computationally efficient reducing the computational complexity by a factor of $\mathcal{O}(N)$, additionally from the algorithms in [12-14]. The authors also 
introduced a method to exploit the conjugate symmetry to enlarge the effective array aperture, which resulted in reasonably impressive performance. However, despite the fact that the performance of the algorithm [15] was reported to outperform that of [14], its performance is still inferior to computationally more expensive MUSIC scheme particularly at low signal-to-noise ratio (SNR). Moreover, based on the cross-correlation matrix between data collected by two component ULAs of L-shaped array, it cannot be used for a single ULA.

In this paper, we propose a low complexity subspacebased DOA estimation algorithm employing direct signal space construction method (DSPCM) similar to that in [15] but by subsampling the autocorrelation matrix of a ULA. The use of autocorrelation matrix in signal space construction enables us to use the low complexity method with a single ULA and to exploit the Toeplitz structure of the autocorrelation matrix in improving the accuracy of estimating signal vectors. We further propose a method of signal vector separation to improve the distinguishability of the signal vectors and hence to improve the performance particularly in the low SNR regime. Finally, we derive root finding (RF) method in addition to the spectral search (SS) method as the angle finding scheme.

The rest of this paper is organized as follows. In Section 2, the system model is described together with the definition of the notations used throughout this paper. In Section 3, the theoretical background of the algorithm is provided. In particular, a sufficient set of conditions are provided for the applicability of the algorithm. Next, the issues of practical algorithm implementation are discussed in Section 4. In this section, we provide two realizations, namely, SS and RF schemes of the proposed DSPCM. Then, the performance of the proposed scheme is compared with existing schemes, particularly with the MUSIC-based schemes and with that proposed in [15], and the computational complexity is analyzed in Section 5. Finally, we draw conclusions and discuss the directions of future work in Section 6.

Notation. The set of complex numbers will be denoted by $\mathbb{C}$ and the set of all $m \times n$ complex matrices will be denoted by $\mathbb{C}^{m \times n}$. The complex conjugate, the transpose, and the conjugate transpose of matrix $A$ are denoted, respectively, by $A^{*}, A^{T}$, and $A^{H}$. Boldface letters are used for matrices and vectors. We will denote by $\left[\mathbf{r}_{1}, \mathbf{r}_{2}, \ldots, \mathbf{r}_{n}\right]$ the matrix whose 1 st, 2 nd, ..., and $n$th columns are $\mathbf{r}_{1}, \mathbf{r}_{2}, \ldots$, and $\mathbf{r}_{m}$, respectively. $n \times n$ identity matrix and $m \times n$ zero matrix are denoted, respectively, by $\mathbf{I}_{n}$ and $\mathbf{O}_{m \times n}$. We will denote by $\delta_{m n}$ the Dirac delta function. For a real number $x,\lfloor x\rfloor$ denotes the largest integer that does not exceed $x$.

\section{System Model}

In this paper, we consider a uniform linear array (ULA) and propose a low complexity subspace-based direction-ofarrival (DOA) estimation algorithm based on correlation matrix subsampling. We assume that $N$ antenna elements are located along the $x$-axis at coordinates $0, d, 2 d, \ldots,(N-1) d$ as shown in Figure 1. We assume that $K$ narrowband far-field

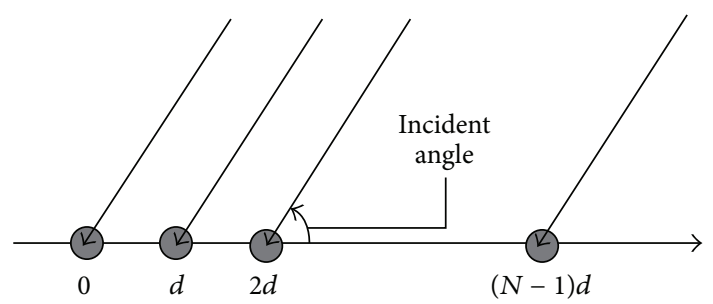

FIGURE 1: Uniform linear array model in this paper.

plane waves of wavelength $\lambda$ impinge on the array making angles $\theta_{1}, \ldots, \theta_{K}$ with the $x$-axis. We let $s_{1}(t), \ldots, s_{K}(t)$ denote the signals corresponding to these $K$ waves arriving at the origin, namely, at the leftmost antenna element in Figure 1. We denote by $x_{1}(t), x_{2}(t), \ldots, x_{N}(t)$ the data collected by the antenna elements located at $x=0, d, \ldots,(N-1) d$, respectively. For notational convenience, we let

$$
\nu_{k}=2 \pi \frac{d \cos \theta_{k}}{\lambda}, \quad k=1,2, \ldots, K .
$$

Here, we assume that $s_{1}(t), \ldots, s_{K}(t), x_{1}(t), \ldots, x_{N}(t)$ represent the complex baseband representation of the relevant signals at time $t$. For compact mathematical description, we let $\mathbf{x}(t)=\left[x_{1}(t), x_{2}(t), \ldots, x_{N}(t)\right]^{T}$ and $\mathbf{s}(t)=$ $\left[s_{1}(t), s_{2}(t), \ldots, s_{K}(t)\right]^{T}$. Then, the system can be modeled by

$$
\mathbf{x}(t)=\mathbf{A s}(t)+\mathbf{n}(t),
$$

where $\mathbf{n}(t)$ represent the additive Gaussian noise vector and A is the $N \times K$ dimensional array response matrix whose $(n, k)$ element is given by $e^{j(n-1) v_{k}}$. We assume that the signal wavelength $\lambda$ is known a priori and that the number $K$ of impinging signals is predetermined by appropriate methods such as that in [16].

We assume that $\mathbf{s}(t)$ and $\mathbf{n}(s)$ are statistically independent complex random vector processes with zero mean satisfying

$$
\begin{aligned}
E\left[\mathbf{s}(t) \mathbf{s}(s)^{H}\right] & =\delta_{t s} \mathbf{P}, \\
E\left[\mathbf{s}(t) \mathbf{s}(s)^{T}\right] & =\mathbf{O}_{K \times K}, \\
E\left[\mathbf{n}(t) \mathbf{n}(s)^{H}\right] & =N_{0} \delta_{t s} \mathbf{I}_{N}, \\
E\left[\mathbf{n}(t) \mathbf{n}(s)^{T}\right] & =\mathbf{O}_{N \times N},
\end{aligned}
$$

where $N_{0}$ is the one-sided noise power spectral density and $\mathbf{P}$ is a positive definite matrix. Throughout this paper, we assume that sources $s_{k}(t)$ 's are statistically uncorrelated so that $\mathbf{P}$ is a diagonal matrix with positive diagonal elements $p_{1}, p_{2}, \ldots, p_{K}$.

\section{Direct Signal Space Construction by Autocorrelation Matrix Subsampling}

In [15], a subspace-based DOA estimation algorithm using the cross-correlation matrix between two data vectors collected by the component ULAs of an L-shaped array has been 
proposed that does not require computationally expensive ED or SVD in constructing the signal or noise subspace. In this paper, we show that similar signal space construction is possible by subsampling the autocorrelation matrix of a ULA with significantly improved performance.

First, we note that the autocorrelation matrix of $\mathbf{x}(t)$ is given by

$$
\mathbf{R} \equiv E\left[\mathbf{x}(t) \mathbf{x}(t)^{H}\right]=\mathbf{A P A}^{H}+N_{0} \mathbf{I}_{N}
$$

In particular, the autocorrelation matrix $\mathbf{R}$ is a Hermitian Toeplitz matrix with its $(m, n)$ element given by

$$
R_{m n}=\rho_{m-n}+N_{0} \delta_{m n}
$$

where

$$
\rho_{m-n}=\sum_{k=1}^{K} p_{k} e^{j(m-n) v_{k}} .
$$

Consequently, $R_{m n}$ does not involve noise component except when $m=n$. In particular, we note that $R_{m n}=\rho_{m-n}$ for any $(m, n)$ with $m \neq n$ values. Noting this fact, we propose methods to construct the signal space by subsampling using only the nondiagonal elements of $\mathbf{R}$.

To be more precise, to construct $K$ dimensional signal space, we define $K L$-dimensional column vectors by

$$
\mathbf{r}_{k}=\left[\begin{array}{c}
\rho_{i_{k}} \\
\rho_{i_{k}+\delta} \\
\rho_{i_{k}+2 \delta} \\
\vdots \\
\rho_{i_{k}+(L-1) \delta}
\end{array}\right], \quad k=1,2, \ldots, K .
$$

Here, we assume that the integers $i_{1}, i_{2}, \ldots, i_{K}, \delta$, and $L$ are chosen to satisfy the following four conditions:

(1) $L$ is larger than $K$.

(2) $\delta$ is larger than 1 .

(3) $-N+1 \leq i_{1}<i_{2}<\cdots<i_{K}<(N-1)-(L-1) \delta$.

(4) None of the $K L$ indices $i_{1}, i_{1}+\delta, \ldots, i_{1}+(L-$ 1) $\delta, \ldots, i_{K}, i_{K}+\delta, \ldots, i_{K}+(L-1) \delta$ is zero.

For illustration of the choice of the indices, let us consider the case of $\delta=2$, which is, in fact, of our primary interest. In this case, we might define

$$
\begin{gathered}
\mathbf{r}_{1}=\left[\rho_{-2 H+1}, \rho_{-2 H+3}, \ldots, \rho_{2 H-2 K+1}\right]^{T} \\
\mathbf{r}_{2}=\left[\rho_{-2 H+3}, \rho_{-2 H+5}, \ldots, \rho_{2 H-2 K+3}\right]^{T} \\
\vdots \\
\mathbf{r}_{K}=\left[\rho_{-2 H+(2 K-1)}, \rho_{-2 H+(2 K+1)}, \ldots, \rho_{2 H-1}\right]^{T},
\end{gathered}
$$

where $H$ denotes the integer $\lfloor N / 2\rfloor$, namely, the largest integer not exceeding $N / 2$.
Next, we define the signal space $\mathcal{S}$ as the $K$ dimensional subspace of $\mathbb{C}^{L \times 1}$ spanned by $\mathbf{r}_{1}, \mathbf{r}_{2}, \ldots, \mathbf{r}_{K}$. To understand the structure of the signal space $\mathcal{S}$, we first note that

$$
\mathbf{r}_{k}=\mathbf{B P} \mathbf{q}_{k} \text {, }
$$

where $\mathbf{B}$ is $L \times K$ matrix given by

$$
\mathbf{B}=\left[\begin{array}{cccc}
1 & 1 & \cdots & 1 \\
e^{j \delta v_{1}} & e^{j \delta v_{2}} & \cdots & e^{j \delta v_{K}} \\
e^{j 2 \delta v_{1}} & e^{j 2 \delta v_{2}} & \cdots & e^{j 2 \delta v_{K}} \\
\vdots & \vdots & \vdots & \vdots \\
e^{j(L-1) \delta v_{1}} & e^{j(L-1) \delta v_{2}} & \cdots & e^{j(L-1) \delta v_{K}}
\end{array}\right]
$$

and $\mathbf{q}=\left[e^{j i_{k} \nu_{1}}, e^{j i_{k} \nu_{2}}, \ldots, e^{j i_{k} \nu_{K}}\right]^{T}$. We recall that the matrix $\mathbf{P}$ is the diagonal matrix with diagonal elements $p_{1}, p_{2}, \ldots, p_{K}$ introduced in Section 2. Consequently, the following relation holds:

$$
\left[\mathbf{r}_{1}, \mathbf{r}_{2}, \ldots, \mathbf{r}_{k}\right]=\mathbf{B P Q}
$$

where $\mathbf{Q}$ is $K \times K$ matrix given by

$$
\left[\begin{array}{cccc}
e^{j i_{1} v_{1}} & e^{j i_{2} v_{1}} & \cdots & e^{j i_{K} v_{1}} \\
e^{j i_{1} \nu_{2}} & e^{j i_{2} v_{2}} & \cdots & e^{j i_{K} v_{2}} \\
\vdots & \vdots & \vdots & \vdots \\
e^{j i_{1} \nu_{K}} & e^{j i_{2} \nu_{K}} & \cdots & e^{j i_{K} \nu_{K}}
\end{array}\right] .
$$

Here, we note that the matrix $\mathbf{B}$ contains the information about the directions of arrivals, namely, $v_{1}, v_{2}, \ldots, v_{K}$. Moreover, if $\mathbf{Q}$ is invertible, we have

$$
\mathbf{B}=\left[\mathbf{r}_{1}, \mathbf{r}_{2}, \ldots, \mathbf{r}_{k}\right] \mathbf{Q}^{-1} \mathbf{P}^{-1}
$$

since $\mathbf{P}$ has already been assumed to be invertible. This means that the columns of $\mathbf{B}$ containing the information about the directions of arrivals can be obtained as a linear combination of $\mathbf{r}_{1}, \mathbf{r}_{2}, \ldots$, and $\mathbf{r}_{K}$. Consequently, the subspace $\mathcal{S}$ generated by $\mathbf{r}_{1}, \mathbf{r}_{2}, \ldots$, and $\mathbf{r}_{K}$ can play the role of signal space if $\mathbf{Q}$ is invertible.

It is easy to note that the Vandermonde matrix $\mathbf{B}$ has rank $K$ if and only if $e^{j \delta v_{1}}, e^{j \delta v_{2}}, \ldots, e^{j \delta v_{K}}$ are all distinct. However, the rank of the matrix $\mathbf{Q}$, which is a generalized Vandermonde matrix, is not easily determined in the most general case. However, if we specialize in the case of $i_{2}-i_{1}=$ $i_{3}-i_{2}=\cdots=i_{K}-i_{K-1}$, which is of particular practical importance, the determinant of $\mathbf{Q}$ is given by

$$
e^{j i_{1} \sum_{k=1}^{K} v_{k}} \prod_{1 \leq k<l \leq K}\left[e^{j\left(i_{2}-i_{1}\right) v_{l}}-e^{j\left(i_{2}-i_{1}\right) v_{k}}\right]
$$

which is nonzero if and only if $e^{j\left(i_{2}-i_{1}\right) v_{1}}, e^{j\left(i_{2}-i_{1}\right) v_{2}}$, $\ldots, e^{j\left(i_{2}-i_{1}\right) \nu_{K}}$ are all distinct. For this reason, we further assume, in what follows, that $\delta$ and $i_{1}, i_{2}, \ldots, i_{K}$ are so chosen that the following three properties hold: 
(1) $i_{2}-i_{1}=i_{3}-i_{2}=\cdots=i_{K}-i_{K-1} \equiv \Delta$,

(2) $e^{j \delta \nu_{1}}, e^{j \delta v_{2}}, \ldots, e^{j \delta \nu_{K}}$ are all distinct,

(3) $e^{j\left(i_{2}-i_{1}\right) v_{1}}, e^{j\left(i_{2}-i_{1}\right) v_{2}}, \ldots, e^{j\left(i_{2}-i_{1}\right) \nu_{K}}$ are all distinct.

Here, the constant $\Delta$ will be referred to as the signal vector separation index. We note that the overlap among signal vectors can be reduced by increasing the value of $\Delta$. Even though the signal space dimension $L$ is decreased, the increase in the value of $\Delta$ can improve the distinguishability among the signal vectors and result in improved performance. We will call this technique signal vector separation.

Now, let us consider $L$ dimensional steering vector $\mathbf{b}(\omega)$ defined by

$$
\mathbf{b}(\omega)=\left[1, e^{j \omega}, e^{j 2 \omega}, \ldots, e^{j(L-1) \omega}\right]^{T} .
$$

Then, just as in ordinary subspace-based schemes such as the MUSIC scheme [17], the following four statements become equivalent:

(1) The vector $\mathbf{b}(\omega)$ belongs to $\mathcal{S}$.

(2) The orthogonal projection $\mathbf{b}(\omega)_{\|}$of $\mathbf{b}(\omega)$ onto $\mathcal{S}$ equals $\mathbf{b}(\omega)$ itself.

(3) The augmented matrix $[\mathbf{B}, \mathbf{b}(\omega)]$, which is a Vandermonde matrix, has rank $K$.

(4) The complex number $e^{j \omega}$ equals one of the $K$ complex numbers $e^{j \delta v_{1}}, e^{j \delta v_{2}}, \ldots, e^{j \delta v_{K}}$.

For the realization of reduced complexity algorithms, we particularly note the equivalence between second and fourth statements. To effectively use this equivalence, we let $\widehat{\mathbf{r}}_{1}, \ldots, \widehat{\mathbf{r}}_{K}$ denote the orthonormal vectors obtained by applying the Gram-Schmidt orthonormalization process on the $K$ vectors $\mathbf{r}_{1}, \mathbf{r}_{2}, \ldots, \mathbf{r}_{K}$. Before proceeding, we note that the Gram-Schmidt orthonormalization can be carried out efficiently via matrix QR-decomposition without requiring computationally expensive ED or SVD process. Since the orthogonal projection $\mathbf{b}(\omega)_{\|}$of $\mathbf{b}(\omega)$ is given by

$$
\mathbf{b}(\omega)_{\|}=\sum_{k=1}^{K}\left[\widehat{\mathbf{r}}_{k}^{H} \mathbf{b}(\omega)\right] \widehat{\mathbf{r}}_{k},
$$

it follows that

$$
\left\|\mathbf{b}(\omega)_{\|}\right\|^{2}=\sum_{k=1}^{K}\left|\widehat{\mathbf{r}}_{k}^{H} \mathbf{b}(\omega)\right|^{2}
$$

But since $\left\|\mathbf{b}(\omega)_{\|}\right\|^{2} \leq\|\mathbf{b}(\omega)\|^{2}$ with equality if and only if $\mathbf{b}(\omega)_{\|}=\mathbf{b}(\omega)$, the following theorem holds.

Theorem 1. The complex number $e^{j \omega}$ equals one of $e^{j \delta v_{1}}, e^{j \delta v_{2}}, \ldots, e^{j \delta v_{K}}$ if and only if

$$
\sum_{k=1}^{K}\left|\widehat{\mathbf{r}}_{k}^{H} \mathbf{b}(\omega)\right|^{2}
$$

takes its maximum possible value $K=\|\mathbf{b}(\omega)\|^{2}$.

\section{DOA Estimation Algorithm Implementation}

4.1. Signal Space Construction and Correlation Estimation. In this paper, we estimate the directions of arrivals using Theorem 1 . We note that the direction information is stored in the signal space basis vectors $\mathbf{r}_{1}, \mathbf{r}_{2}, \ldots, \mathbf{r}_{K}$ or, equivalently, in the orthogonal basis vectors $\widehat{\mathbf{r}}_{1}, \widehat{\mathbf{r}}_{2}, \ldots, \widehat{\mathbf{r}}_{K}$. We recall that the vectors $\mathbf{r}_{k}$ 's are constructed using nondiagonal elements of the correlation matrix $\mathbf{R}$. Consequently, we only need to estimate the values of $\rho_{l}$ 's for nonzero $l$ values. Before further proceeding, we note that $\rho_{-l}=\rho_{l}^{*}$. Consequently, we only need to estimate the values of $\rho_{l}$ 's for positive $l$ values.

To discuss how we obtain $\rho_{k}$, we first note $\rho_{l}=R_{l+11}=$ $R_{l+22}=\cdots=R_{N N-l}$ for $l=1,2, \ldots, N-1$. As in most subspace-based schemes, the estimate $\widehat{R}_{m n}$ of $R_{m n}$ can be obtained by

$$
\widehat{R}_{m n}=\frac{1}{T} \sum_{t=1}^{T} x_{m}(t) x_{n}^{*}(t)
$$

Based on this estimate of $R_{m n}$, we can obtain the estimates of $\rho_{l}$ 's with varying degree of accuracy and computational complexity. The first and simplest way is to use one of $\widehat{R}_{l+11}, \widehat{R}_{l+22}, \ldots, \widehat{R}_{N N-l}$ as the estimate $\rho_{l}^{(1)}$ of $\rho_{l}$. Instead of this estimate that poorly exploits the Toeplitz structure, we may well employ an averaging method to obtain the improved estimates $\rho_{l}^{(2)}$ defined by

$$
\rho_{l}^{(2)}=\frac{1}{N-l} \sum_{m=l+1}^{N} \widehat{R}_{m m-l}
$$

for $l=1,2, \ldots, N-1$. Although these second estimates greatly improve the estimation accuracy over the first estimates $\rho_{l}^{(1)}$, s, they require more computational burden. Consequently, we also consider a partial averaging method to improve the accuracy with limited increase in computational complexity. To be more specific, we define the third estimates $\rho_{l}^{(3)}, l=$ $1,2, \ldots, N-1$ by

$$
\rho_{l}^{(3)}=\frac{1}{M \cdot T} \sum_{m=N-M+1}^{N} \sum_{t=1}^{T} x_{m}(t) x_{m-l}^{*}(t),
$$

if $l \leq N-M$, and by

$$
\rho_{l}^{(3)}=\frac{1}{(N-l) \cdot T} \sum_{m=l+1}^{N} \sum_{t=1}^{T} x_{m}(t) x_{m-l}^{*}(t),
$$

if $l \geq N-M$, with a positive integer $M$ suitably chosen depending on the situation at hand.

4.2. Choice of Parameters. Once we obtain the estimates of $\rho_{l}$ 's, we can readily construct the orthonormal basis vectors $\widehat{\mathbf{r}}_{1}, \ldots, \widehat{\mathbf{r}}_{K}$ of the signal space $\mathcal{S}$. However, we need to choose the right values for the parameters $\delta, i_{1}, \Delta, L$, and $M$ to achieve optimal performance in a computationally efficient way. The DOA estimation in this paper will be carried out by equating $e^{j \omega}$ with one of $e^{j 2 \pi \delta d \cos \theta_{1} / \lambda}, e^{j 2 \pi \delta d \cos \theta_{2} / \lambda}, \ldots, e^{j 2 \pi \delta d \cos \theta_{K} / \lambda}$ 
using Theorem 1. However, the exponential function $e^{j x}$ exhibits periodicity with period $2 \pi$. Hence, we need to choose the values of $\delta$ and $d$ to satisfy

$$
\delta d \leq \frac{\lambda}{2}
$$

to be able to identify uniquely the values of $\theta_{1}, \theta_{2}, \ldots, \theta_{K}$ without any prior information about their values. To increase the array aperture size and hence to improve the resolution we need to choose the value of $\lambda$ as large as possible. Consequently, we usually put $\delta=2$ and $d=\lambda / 4$ for best estimation accuracy.

Next, we discuss the choice of the parameters $i_{1}, \Delta$, and $L$ for given value of $\delta$. We can achieve maximum utilization of the elements of $\mathbf{R}$ if we put $i_{1}=-N+1, \Delta=\delta$, and $L=\lfloor(2 N-K-1) / \delta\rfloor+1$. This is a reasonable choice for most situations since we can generally achieve better estimation accuracy with increased effective observation space dimension $L$. However, we note that there is significant overlap between $\mathbf{r}_{k}$ and $\mathbf{r}_{k+1}$, which can make the signal vectors $\mathbf{r}_{k}$ 's indistinguishable when the signal-to-noise ratio (SNR) is low. One possible way to reduce the overlap among the signal vectors $\mathbf{r}_{k}$ 's is to choose the signal vector separation index $\Delta$ large. However, if we choose a large value for $\Delta$, it will decrease the effective observation space dimension $L$, which can then reduce the resolution. Consequently, it is very important to choose an appropriate value for $\Delta$. We also note that the estimates of $\rho_{l}$ 's are more accurate for small $|l|$ values and less accurate for large $|l|$ values if we adopt $\rho_{l}^{(2)}$ or $\rho_{l}^{(3)}$ for the estimation of $\rho_{l}$. Consequently, we may avoid using $\rho_{l}$ 's with large $|l|$ values, which is particularly useful when the number $N$ of antenna elements is large. If we avoid using $\rho_{-N+1}, \ldots, \rho_{-N+m-1}$ and $\rho_{N-m+1}, \ldots, \rho_{N-1}$, the maximum possible effective observation space dimension is given by $L=\lfloor(2 N-2 m-(K-1) \Delta) / \delta\rfloor+1$.

4.3. DOA Estimation Algorithms: Spectral Search and Root Finding. Once we have chosen the parameters $\delta, i_{1}, \Delta, L$, and $M$ appropriately as discussed in the previous subsection, we can apply Theorem 1 to determine the directions of arrivals. As usual, the directions of arrivals can be obtained by spectral search directly applying Theorem 1 . This method is suitable when the values of $\theta_{k}$ 's are already estimated and only additional tracking or finer estimation is necessary. However, the spectral search method may prove to be computationally expensive for initial estimation. To avoid the burden of spectral search, we can also adopt root finding method with slight modification on the usual method.

To discuss how the root finding scheme can be derived, let us note that the spectral search method searches for $\omega$ that satisfy

$$
\mathbf{b}(\omega)^{H}\left[\sum_{k=1}^{K} \widehat{\mathbf{r}}_{k} \widehat{\mathbf{r}}_{k}^{H}\right] \mathbf{b}(\omega)=K .
$$

From (24), we note that we can alternatively search for unitnorm zeros of the following equations:

$$
\mathbf{b}\left(\frac{1}{z}\right)^{H}\left[\sum_{k=1}^{K} \widehat{\mathbf{r}}_{k} \widehat{\mathbf{r}}_{k}^{H}\right] \mathbf{b}(z)=K,
$$

obtaining the root finding realization of the proposed algorithm. We note that the matrix $\sum_{k=1}^{K} \widehat{\mathbf{r}}_{k} \widehat{\mathbf{r}}_{k}^{H}$ is Hermitian and hence $1 / z^{*}$ is also a zero of (25) if $z$ is a zero of (25).

\section{Performance and Computational Complexity}

5.1. Performance Evaluation. In this section, we compare the performance of the proposed algorithm with existing schemes. As the measure of performance, we use the root mean squared error (RMSE) defined by

$$
\mathrm{RMSE}=\sqrt{\sum_{k=1}^{K} E\left[\left|\widehat{\theta}_{k}-\theta_{k}\right|^{2}\right]}
$$

where $\hat{\theta}_{k}$ denotes the estimate of $\theta_{k}$. In what follows, the angles and RMSE will be measured in degrees rather than radians.

For performance evaluation, we first consider the situation in which $K=4$ equal strength uncorrelated narrowband signals arrive on a ULA with $N=20$ antenna elements separated by $d=\lambda / 4$. We assume that the incident angles are $\theta_{1}=75^{\circ}, \theta_{2}=85^{\circ}, \theta_{3}=95^{\circ}$, and $\theta_{4}=105^{\circ}$. We assume that $T=100$ and that the second estimate $\rho_{l}^{(2)}$ is used to obtain $\rho_{l}$ unless stated otherwise. While we have developed the proposed algorithm valid for any integer $\delta$ value greater than or equal to 2 , we will assume that $\delta$ is chosen to be 2 in what follows to discuss various issues in a space-effective manner. We will also assume that $i_{1}$ is chosen to be $-N+1$ in what follows. We first study the impact of the choice of the signal vector separation index $\Delta$ on the performance. To study the trade-off of choosing different $\Delta$ values, we consider 2,4 , and 6 for the value of $\Delta$. (We note that we cannot avoid using $\rho_{0}$ if we choose an odd number for $\Delta$.) For these $\Delta$ values, the maximum possible effective observation space dimension is given by 17,14 , and 11 , respectively. We have chosen these $L$ values in our simulations, the results of which are illustrated in Figure 2. For the simulations, we considered both spectrum search (SS) and root finding (RF) methods in implementing the proposed algorithm. In Figure 2, we clearly see that the different choice of $\Delta$ value can result in significant difference in the performance. In particular, we note that the performance is significantly improved by increasing $\Delta$ from 2 to 4 , although the performance is degraded if we further increase $\Delta$ to 6 . Consequently, Figure 2 confirms the importance of reducing the overlap between signal space vectors by appropriately separating the elements. To see more intuitively how the choice of $\Delta$ value affects the performance, 


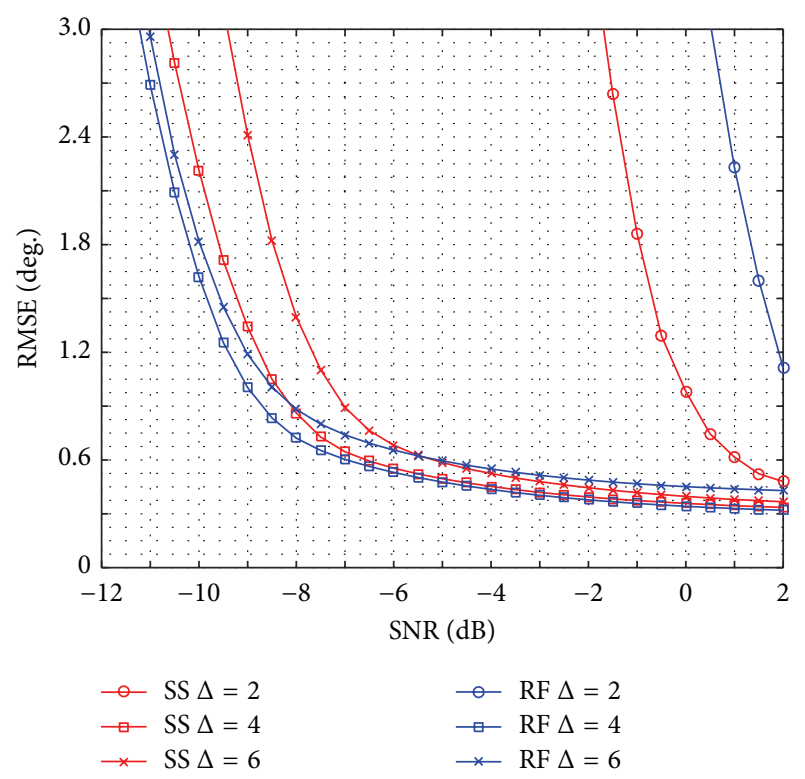

FIGURE 2: Signal vector separation $\Delta$ and the performance of proposed scheme. Results with four signals arriving at angles $75^{\circ}$, $85^{\circ}, 95^{\circ}$, and $105^{\circ}$.

we include two figures, namely, Figures 3 and 4 , which depict the value $f(\theta)$ defined by

$$
f(\theta)=\frac{1}{K-\sum_{k=1}^{K}\left|\widehat{\mathbf{r}}_{k}^{H} \mathbf{b}(\omega)\right|^{2}}
$$

with $\omega=e^{j 2 \pi \delta \cos \theta / \lambda}$. Figure 3 depicts the results with $\Delta=$ 2 at three different signal-to-noise ratios, while Figure 4 represents those with $\Delta=4$. To draw each of these figures, we randomly generated signals according to the specified average signal-to-noise ratio and the results are plotted as a function of the incident angle $\theta$. We clearly see that the peak values are much more easily recognizable with $\Delta=4$ than with $\Delta=2$ at the same signal-to-noise ratio. Before proceeding, we also note that the root finding method provides slightly lower RMSE values (except when $\Delta=1$ ) in comparison with spectral search method, as in the case of MUSIC schemes $[6,18]$.

In obtaining the results given in Figure 2, we used the second estimate $\rho_{l}^{(2)}$ to obtain $\rho_{l}$. However, the computation of $\rho^{(2)}$ can be burdensome in some situations. For this reason, we propose using $\rho_{l}^{(3)}$ in such situations with appropriately chosen $M$ values. To see how well we can approach, with reduced complexity, we consider the above system setup with $\Delta=4$. The results of performance evaluation are given in Figure 5. We note that the second estimate $\rho_{l}^{(2)}$ reduces to the first and third estimates $\rho_{l}^{(1)}$ and $\rho_{l}^{(3)}$, respectively, at the extreme cases of $M=1$ and $M=19$. In Figure 5, we observe that the performance is enhanced with increased $M$ values. We also note that the performance enhancement is particularly conspicuous when $M$ is increased from a small value. For example, by increasing $M$ from 1 to 3 or 5, we can achieve about $3.5 \mathrm{~dB}$ or $5.0 \mathrm{~dB}$ gain, respectively, at RMSE

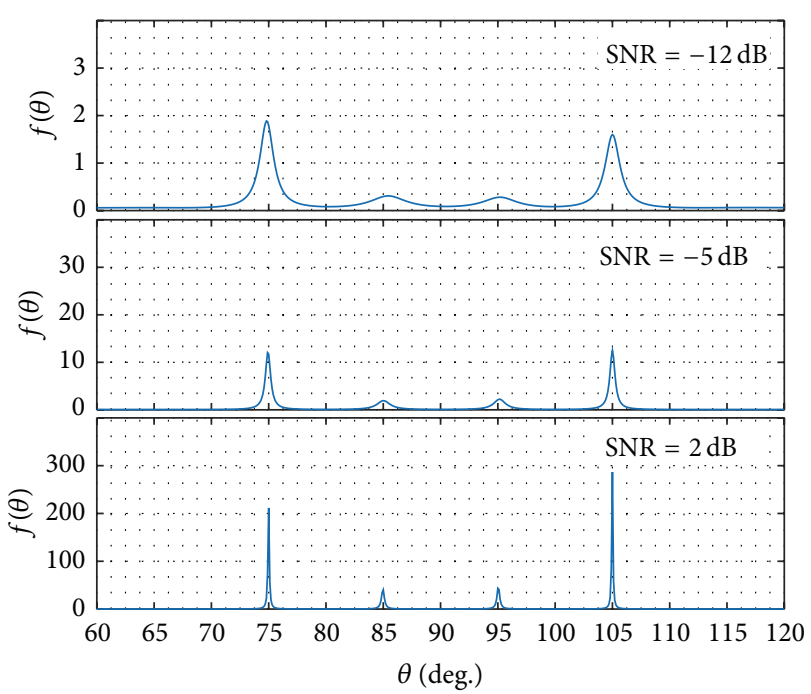

Figure 3: Sample $f(\theta)$ values with $N=20, K=4, \Delta=2, L=17$. Results with signals arriving at angles $75^{\circ}, 85^{\circ}, 95^{\circ}$, and $105^{\circ}$.

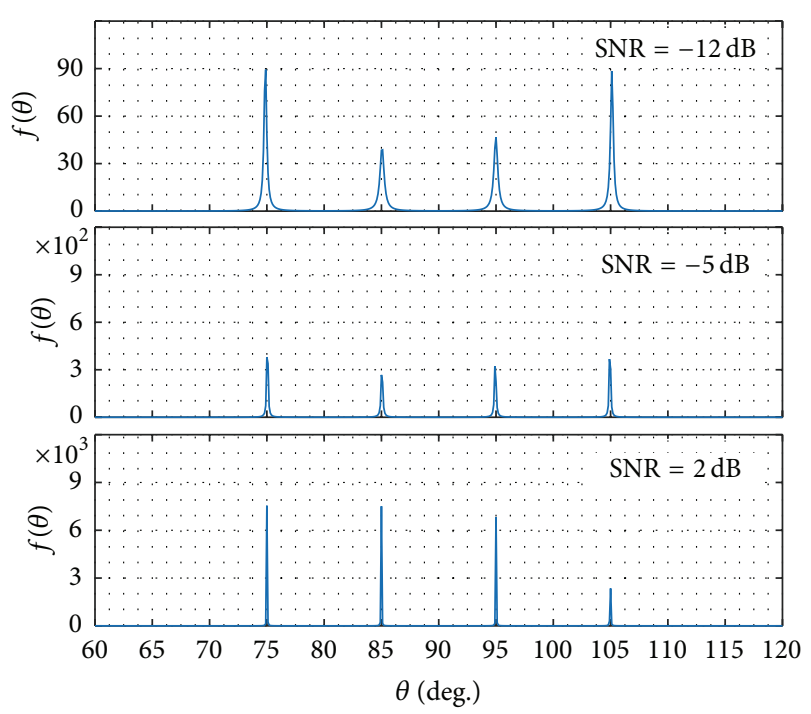

Figure 4: Sample $f(\theta)$ values with $N=20, K=4, \Delta=4, L=14$. Results with signals arriving at angles $75^{\circ}, 85^{\circ}, 95^{\circ}$, and $105^{\circ}$.

value of 0.9 . However, we can achieve only about $1.7 \mathrm{~dB}$ gain by increasing $M$ value from 5 to 19 . Consequently, it is possible to reduce the computational complexity by employing the third estimate $\rho_{l}^{(3)}$ with reasonably small $M$ value without significant performance sacrifice.

Now, we compare the proposed algorithm with existing schemes, particularly with MUSIC-based schemes. The result is given in Figure 6, in which the proposed scheme is referred to as DSPCM standing for direct signal space construction method. In Figure 6, SS DSPCM and RF DSPCM represent the same curves labeled, respectively, as SS $\Delta=4$ and RF $\Delta=4$ in Figure 2. We observe that DSPCM holds good performance against the MUSIC schemes. In Figure 6, MUSIC refers to ordinary MUSIC scheme employing (19) to estimate the covariance matrix, while TMUSIC stands for the MUSIC 


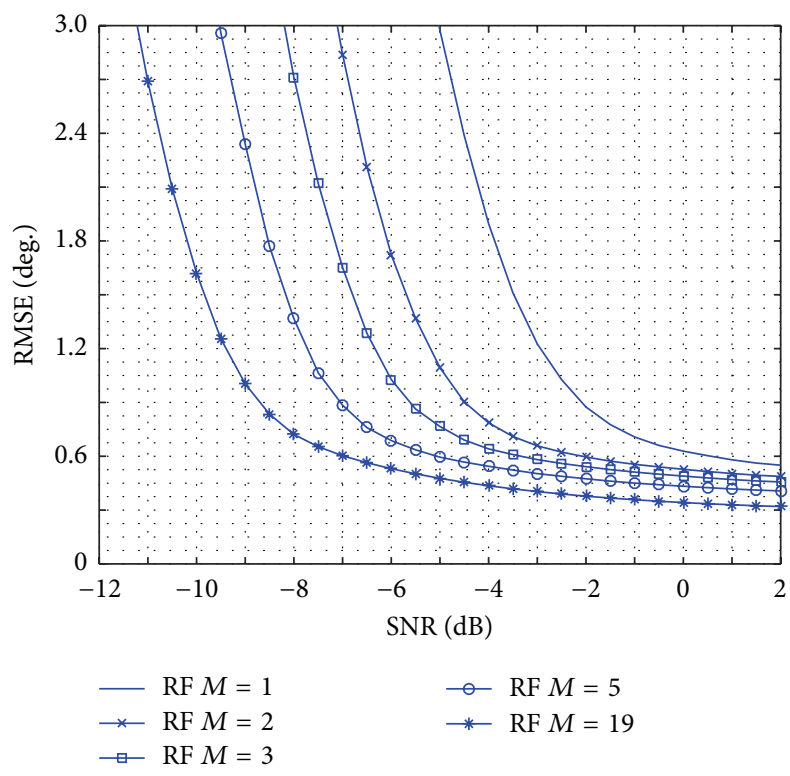

FIGURE 5: Correlation estimation accuracy and the performance of the proposed scheme. The meaning of the constant $M$ is defined in Section 4.1. Results with four signals arriving at angles $75^{\circ}, 85^{\circ}, 95^{\circ}$, and $105^{\circ}$.

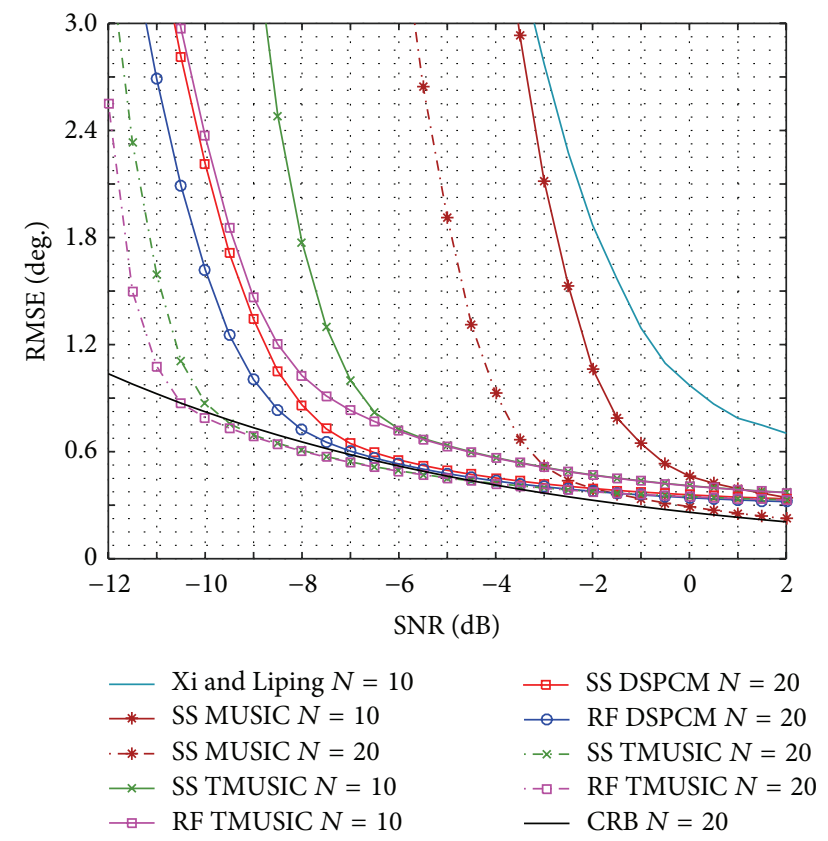

FIGURE 6: Performance of various DOA estimation schemes. Results with four signals arriving at angles $75^{\circ}, 85^{\circ}, 95^{\circ}$, and $105^{\circ}$.

scheme employing the second estimate $\rho_{l}^{(2)}$ to estimate the covariance matrix with Toeplitz property. By comparing SS MUSIC and SS TMUSIC, we observe that the enhanced accuracy with Toeplitz property provides better performance at low SNR. We note that the proposed scheme with $\delta=2$ is based on $N / 2 \times N / 2$ submatrix of the autocorrelation matrix, which is essentially a cross-correlation matrix between two ULAs with $N / 2$ antenna elements with antenna spacing

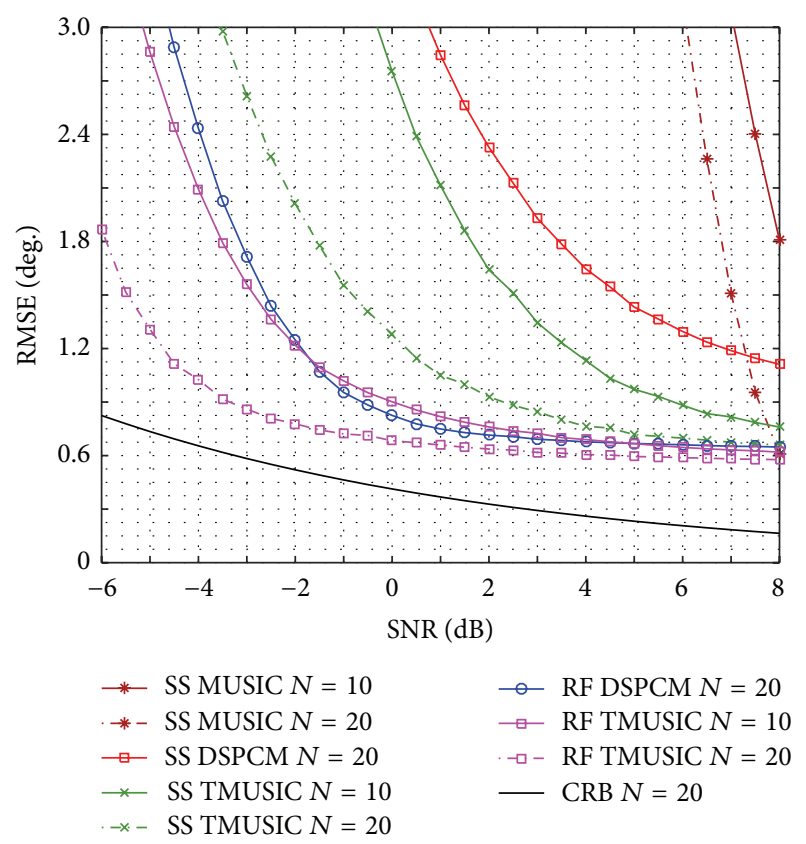

FIGURE 7: Performance of various DOA estimation schemes. Results with four signals arriving at angles $75^{\circ}, 87.5^{\circ}, 92.5^{\circ}$, and $105^{\circ}$.

$2 d$. For this reason, we considered, to compare with the proposed scheme, two antenna configurations for the MUSIC algorithm, one consisting of $N=10$ antenna elements separated by distance $d=\lambda / 2$ and the other consisting of $N=20$ antenna elements separated by distance $d=\lambda / 4$. We note that RF provides better performance (compared with SS) and $N=20$ array configuration leads to better performance (compared with $N=10$ configuration). We have also evaluated the performance of the method proposed in [15]. Other subspace-based algorithms not requiring ED or SVD are compared in [14, 15]. According to [14], the algorithms proposed in [13] and [19] are outperformed by that in [14]. Also, the algorithm proposed in [15] is reported, in [15], to outperform that in [14]. Consequently, we can comfortably conclude that the algorithm proposed in this paper outperforms the algorithms in $[13-15,19]$, since it outperforms that in [15] by a significant margin as illustrated in Figure 6. Finally, we included the RMSE value obtained by Cramer-Rao bound [20]. The performances of the proposed algorithm and TMUSIC scheme are very close to the CRB for SNR greater than $-8 \mathrm{~dB}$. The performance of TMUSIC with $N=20$ even falls slightly below, at certain SNR values, with some small amount of bias induced in the simulations.

Figures 2-6 were obtained assuming that the incident angles are $\theta_{1}=75^{\circ}, \theta_{2}=85^{\circ}, \theta_{3}=95^{\circ}$, and $\theta_{4}=105^{\circ}$. To examine how the proposed scheme performs in more severe situations, we next consider the case in which the incident angles are $\theta_{1}=75^{\circ}, \theta_{2}=87.5^{\circ}, \theta_{3}=92.5^{\circ}$, and $\theta_{4}=105^{\circ}$. The results of performance evaluation are given in Figure 7 . In this case, we clearly see the advantage of having more antenna elements in achieving better resolution. We also note that RF method which allows for zero slightly outside the unit circle is much better suited than the SS method 


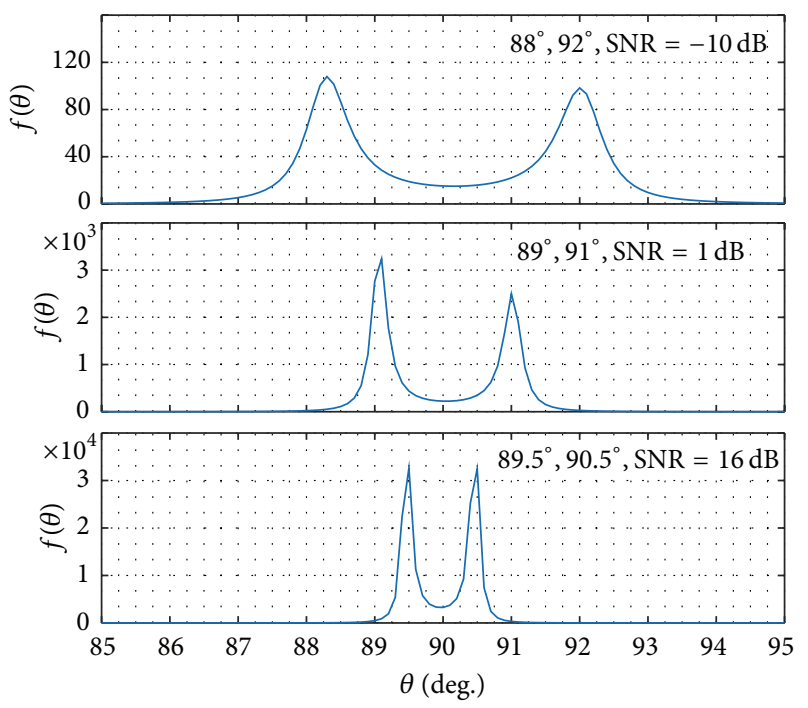

Figure 8: Resolvability of closely spaced incident waves. Sample $f(\theta)$ functions with $N=20, K=2, \Delta=14$.

when two or more incident angles are very closely spaced. Although the performance gap between RF DSPCM and RF MUSIC with $N=20$ becomes wider in Figure 7 than in Figure 6, we believe that the performance of RF DSPCM is still very competitive considering the relative computational simplicity. Unlike in Figure 6, the algorithm proposed in [15] has not been included since its performance cannot be included in the framework. For example, the RMSE values do not fall below $10^{\circ}$ even at SNR of $15 \mathrm{~dB}$. In Figure 7, we note two salient differences in comparison with Figure 6. First, the performance of the proposed scheme as well as that of TMUSIC is not very close to that of CRB. Secondly, the original MUSIC, while inferior to TMUSIC at low SNR, outperforms TMUSIC at very high SNR. From this, we note that the exploitation of Toeplitz property is particularly useful at low SNR.

To further study the resolvability of more closely spaced incident waves, we depicted $f(\theta)$ values with three different scenarios in Figure 8. In each of the three scenarios, it is assumed that $K=2$ incident waves arrive in a ULA with $N=20$ antenna elements and that the value 14 is chosen for the value of $\Delta$. In the first scenario, the incoming waves are assumed to arrive at incident angles at $88^{\circ}$ and $92^{\circ}$. We note that the peak values of $f(\theta)$ are easily identifiable even at very low SNR of $-10 \mathrm{~dB}$. In the next two scenarios, the incident waves are more closely spaced with angle separations $2^{\circ}$ and $1^{\circ}$, respectively. We note that the peaks are again separable even though high signal strength is required in these situations.

Finally, we study the number of antenna elements required to resolve a given number of incident waves. We recall that the dimension $L$ of the reconstructed observation vector is given by $\lfloor(2 N-K-1) / \delta\rfloor+1$. Consequently, to resolve $K$ distinct incident waves, we should have at least $N$ antenna elements satisfying $L=\lfloor(2 N-K-1) / \delta\rfloor+1>K$. In principle, we may choose $N$ values to satisfy $L=K+1$.

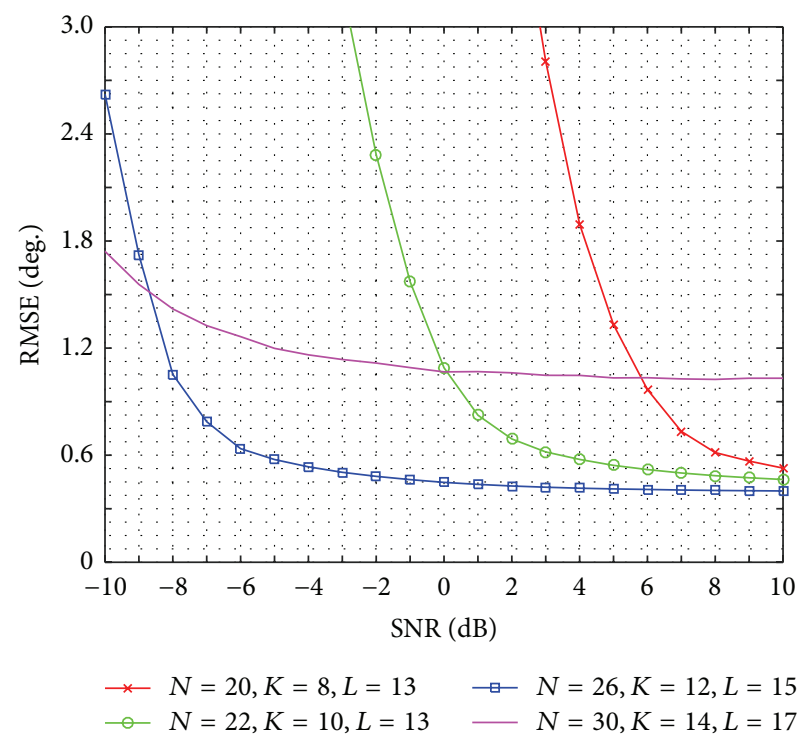

FIGURE 9: Exemplary illustrations of required $N$ values for given $K$ values.

However, according to our evaluations, $L-K$ must be chosen to be no less than 3 to resolve $K$ waves at reasonable SNR values. In Figure 9, we illustrate the simulation results with various $N$ and $K$ values. In each of the results in Figure 9, the value 2 is chosen for the value of $\Delta$ and the incident angles of the $K$ waves are separated by $10^{\circ}$. For example, for $K=8$, the incident angles are assumed to be $55^{\circ}, 65^{\circ}, \ldots, 115^{\circ}$, and $125^{\circ}$. In each of the situations, $N$ is chosen to be such that $L=K+3$ except when $K=8$. From Figure 9 , we see that the choice $L=$ $K+3$ generally provides reasonable estimation performance except for $K=8$. The reason for relatively large required $L-K$ value for $K=8$ is related to the antenna aperture size. For example, even for $K=2$, the choice $N=6$ that yields $L=5$ cannot provide enough antenna aperture size and hence cannot yield excellent performance. Consequently, even for $K=2$, it is generally required to choose $N$ greater than 8.

5.2. Computational Complexity. Three most computationally expensive steps in subspace-based DOA estimation schemes are the estimation of correlation matrix, the acquisition of signal or noise subspace, and the step of finding the DOA angles. In this subsection, we consider only the complexity of the second step, namely, the computational complexity of the signal or noise subspace acquisition step. There are several reasons for this. First of all, the first and third steps are required equally for other subspace-based schemes. Secondly, the complexity of these steps depends heavily on the situations at hand. For example, the first step of estimating the correlation matrix can be computationally burdensome for a single-shot or initial DOA estimation. However, adaptive differential correlation matrix update can be achieved with negligible or at least with reduced complexity in continuous target tracking applications. Similar argument holds true for the case of the third step. For example, the computational complexity of this step can greatly be reduced by adopting 
root finding method instead of spectral search. Moreover, in the case of continuous target tracking, extensive search is not strictly necessary since the target is likely to be in the neighborhood of the previous location. Consequently, we will focus solely on the computational complexity of the subspace acquisition step, which depends only on the algorithm we adopt.

First of all, we note that the proposed algorithm requires only about $K[8 K L-2 L-K]$ real floating point operations (rflops). As long as the number $K$ of targets and the effective observation space dimension $L$ are the same, the algorithm proposed here and the one in [15] require essentially the same computational complexity because similar signal subspace construction methods are used with only slight performance enhancing modifications. We next consider the complexity of subspace acquisition in MUSIC scheme. We recall that eigendecomposition of the correlation matrix is required in MUSIC scheme. The eigendecomposition of the correlation matrix, which is Hermitian, is performed in three steps, in general, to reduce the computational complexity. In the first step, the given Hermitian correlation matrix is converted into a Hermitian tridiagonal matrix. Next, the eigenvalues and eigenvectors of the tridiagonal matrix are obtained through algorithms such as divide-and-conquer or tridiagonal QR iteration method as discussed in [21,22]. Then, the eigenvectors of the original Hermitian matrix are obtained by multiplying a matrix stored during the first step by the eigenvectors of the tridiagonal matrix. Since the computational complexity of the first step is more significant for our applications, we will consider only the complexity of the tridiagonal reduction process. As discussed in Sections 4.4.7 and 5.3.1 of [21], the first step of tridiagonal reduction is usually carried out with Hessenberg reduction process, which requires about $8 N^{3} / 3$ arithmetics if both eigenvalues and eigenvectors of $N$ dimensional symmetric matrix are desired to be found. The same reduction process can be used for a complex Hermitian matrix but with $8 N^{3} / 3$ complex arithmetics, approximately half of which are complex multiplications. We recall that a complex multiplication requires six real arithmetics while a complex addition corresponds to two real arithmetics. Consequently, we can conclude that the eigendecomposition of $N$ dimensional correlation matrix requires at least about $6 \times 4 N^{3} / 3+2 \times 4 N^{3} / 3=32 N^{3} / 3$ rflops, which is significantly larger than $K[8 K L-2 L-K]$ rflops.

For numerical comparison of the computational complexity, let us consider the simulation setups used to produce the results in Figures 6 and 7. We recall that the number $K$ of sources was 4 and that the effective observation space dimension $L$ was 14 for the proposed scheme. Consequently, the proposed DSPCM requires 1,664 rflops. In contrast, the MUSIC schemes require at least about $32 N^{3} / 3=10,667$ and $32 N^{3} / 3=85,333$ rflops, respectively, for $N=10$ and $N=20$ element antenna array setups. Consequently, the MUSIC schemes in Figures 6 and 7 require at least about 6 to 50 times the computational complexity in constructing the signal or noise subspace. In fact, we note that the complexity difference will grow as a function of $\mathcal{O}\left(N^{2} / K\right)$ as the number $N$ of array antenna elements increases, which means more than a thousand times complexity reduction can be achieved when $N \geq 100$.

\section{Conclusion}

In this paper, we proposed a low complexity subspacebased DOA estimation algorithm for a uniform linear array. In the proposed scheme, the signal space is constructed directly, without using computationally expensive ED or SVD process, by subsampling the autocorrelation matrix of a ULA. To avoid possible lack of distinguishability among the constructed signal space basis vectors, particularly at low SNRs, we proposed a signal vector separation method by slightly trading off the signal space dimension. Through simulations, we showed that the signal vector separation method can indeed improve the performance significantly. In addition to spectral search method, we provided root finding scheme as the angle finding methods. We showed that the performance of the proposed scheme is significantly better than the one in [15] almost matching that of the MUSIC schemes under various scenarios. Finally, we illustrated that the computational complexity of the proposed scheme is reduced by a factor of $\mathcal{O}\left(N^{2} / K\right)$ in comparison with MUSIC schemes, where $K$ is the number of sources and $N$ is the number of antenna arrays.

While the proposed algorithm exhibits a number of useful features, there are several issues to be further resolved for its practical use. First, we recall that the computational complexity of subspace-based DOA estimation schemes stems from three computationally burdensome stages, namely, the acquisition of correlation matrix, the construction of subspace, and the final angle finding stage. For this reason, we are planning to develop methods to further reduce the computational burden of correlation matrix acquisition and the final angle finding stage of spectral search or root finding. Next, we will study methods to avoid or reduce the detrimental effect of mutual coupling, since we are dealing with antenna elements spaced more densely than usual and hence the mutual coupling effect can become very critical.

\section{Conflict of Interests}

The author declares that there is no conflict of interests regarding the publication of this paper.

\section{Acknowledgment}

This research was supported by Basic Science Research Program through the National Research Foundation of Korea (NRF) funded by the Ministry of Education (NRF-20100025062 and NRF-2015R1D1A1A01060234).

\section{References}

[1] V. F. Pisarenko, "The retrieval of harmonics from a covariance function," Geophysical Journal International, vol. 33, no. 3, pp. 347-366, 1973. 
[2] G. Bienvenu, "Influence of the spatial coherence of the background noise on high resolution passive methods," in Proceedings of the IEEE International Conference on Acoustics, Speech, and Signal Processing (ICASSP '79), vol. 4, pp. 306-309, IEEE, Washington, DC, USA, April 1979.

[3] R. O. Shcmidt, "Multiple emitter location and signal parameter estimation," in Proceedings of the RADC, Spectral Estimation Workshop, pp. 243-258, Rome, NY, USA, 1979.

[4] R. Kumaresan and D. W. Tufts, "Estimating the angles of arrival of multiple plane waves," IEEE Transactions on Aerospace and Electronic Systems, vol. AES-19, no. 1, pp. 134-139, 1983.

[5] R. Roy and T. Kailath, "ESPRIT-estimation of signal parameters via rotational invariance techniques," IEEE Transactions on Acoustics, Speech, and Signal Processing, vol. 37, no. 7, pp. 984995, 1989.

[6] A. Barabell, "Improving the resolution performance of eigenstructure-based direction-finding algorithms," in Proceedings of the IEEE International Conference on Acoustics, Speech, and Signal Processing (ICASSP '83), vol. 8, pp. 336-339, April 1983.

[7] T. P. Bronez, "Sector interpolation of non-uniform arrays for efficient high resolution bearing estimation," in Proceedings of the International Conference on Acoustics, Speech, and Signal Processing (ICASSP '88), vol. 5, pp. 2885-2888, IEEE, New York, NY, USA, April 1988.

[8] B. Friedlander, "The root-MUSIC algorithm for direction finding with interpolated arrays," Signal Processing, vol. 30, no. 1, pp. 15-29, 1993.

[9] M. A. Doron and E. Doron, "Wavefield modeling and array processing, part I-spatial sampling," IEEE Transactions on Signal Processing, vol. 42, no. 10, pp. 2549-2559, 1994.

[10] T.-J. Shan, M. Wax, and T. Kailath, "On spatial smoothing for direction-of-arrival estimation of coherent signals," IEEE Transactions on Acoustics, Speech and Signal Processing, vol. 33, no. 4, pp. 806-811, 1985.

[11] Q. Y. Yin, R. W. Newcomb, and L. H. Zou, "Estimating 2-D angles of arrival via two parallel linear arrays," in Proceedings of the International Conference on Acoustics, Speech, and Signal Processing (ICASSP '89), vol. 4, pp. 2803-2806, IEEE, Glasgow, Scotland, May 1989.

[12] C.-C. Yeh, "Projection approach to bearing estimation," IEEE Transactions on Acoustics, Speech, and Signal Processing, vol. 34, no. 5, pp. 1347-1349, 1986.

[13] S. Marcos, A. Marsal, and M. Benidir, "The propagator method for source bearing estimation," Signal Processing, vol. 42, no. 2, pp. 121-138, 1995.

[14] G. Wang, J. Xin, N. Zheng, and A. Sano, "Computationally efficient subspace-based method for two-dimensional direction estimation with L-shaped array," IEEE Transactions on Signal Processing, vol. 59, no. 7, pp. 3197-3212, 2011.

[15] N. Xi and L. Liping, "A computationally efficient subspace algorithm for 2-D DOA estimation with L-shaped array," IEEE Signal Processing Letters, vol. 21, no. 8, pp. 971-974, 2014.

[16] M. Wax and T. Kailath, "Detection of signals by information theoretic criteria," IEEE Transactions on Acoustics, Speech, and Signal Processing, vol. 33, no. 2, pp. 387-392, 1985.

[17] R. O. Schmidt, "Multiple emitter location and signal parameter estimation," IEEE Transactions on Antennas and Propagation, vol. 34, no. 3, pp. 276-280, 1986.

[18] B. D. Rao and K. V. S. Hari, "Performance analysis of rootmusic," in Proceedings of the 22nd Asilomar Conference on
Signals, Systems, and Computers, vol. 2, pp. 578-582, Pacific Grove, Calif, USA, 1988.

[19] J. Xin and A. Sano, "Computationally efficient subspace-based method for direction-of-arrival estimation without eigendecomposition," IEEE Transactions on Signal Processing, vol. 52, no. 4, pp. 876-893, 2004.

[20] P. Stoica and A. Nehorai, "MUSIC, maximum likelihood, and cramer-rao bound," IEEE Transactions on Acoustics, Speech, and Signal Processing, vol. 37, no. 5, pp. 720-741, 1989.

[21] J. W. Demmel, Applied Numerical Linear Algebra, SIAM, 1997.

[22] G. H. Golub and C. F. van Loan, Martrix Computations, Johns Hopkins University Press, Baltimore, Md, USA, 4th edition, 2012. 

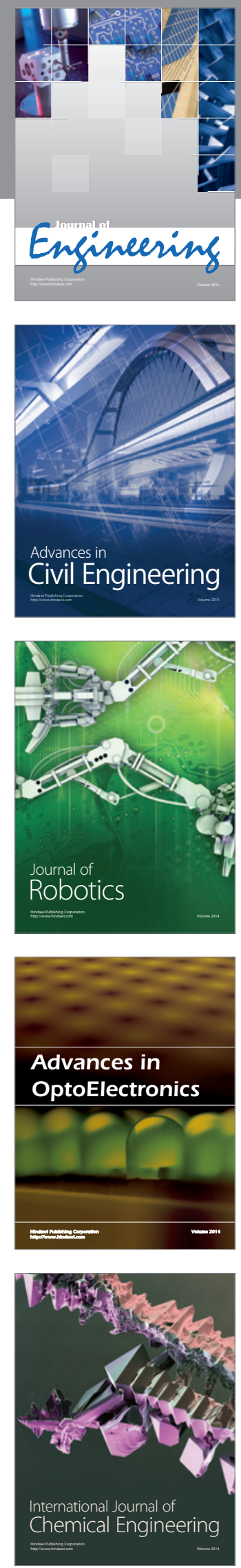

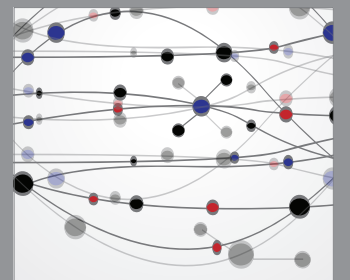

The Scientific World Journal
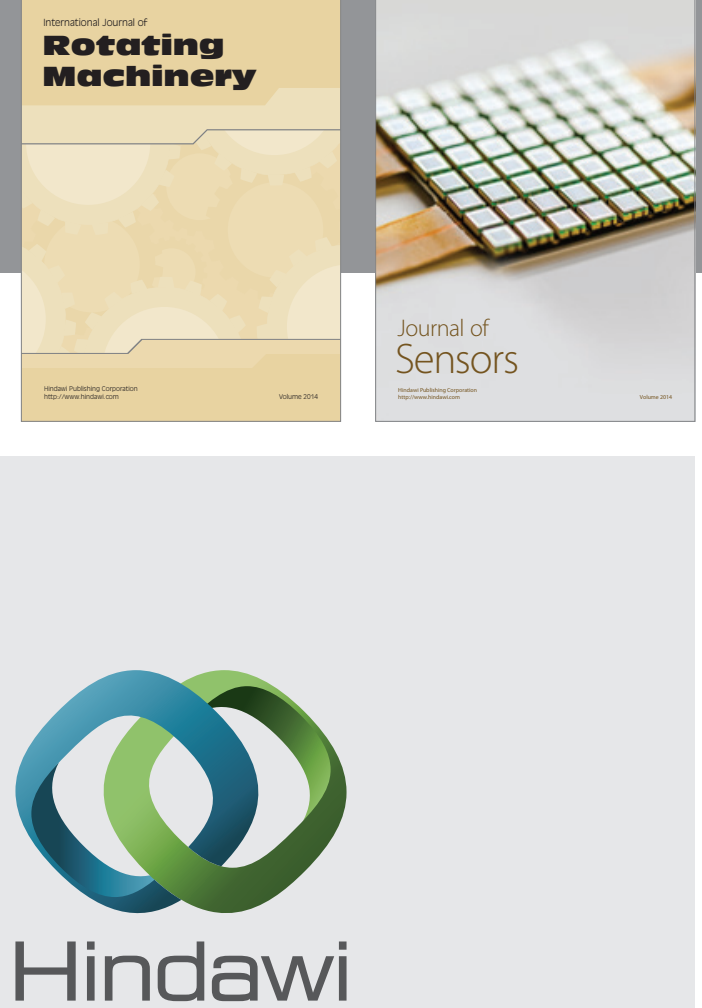

Submit your manuscripts at http://www.hindawi.com
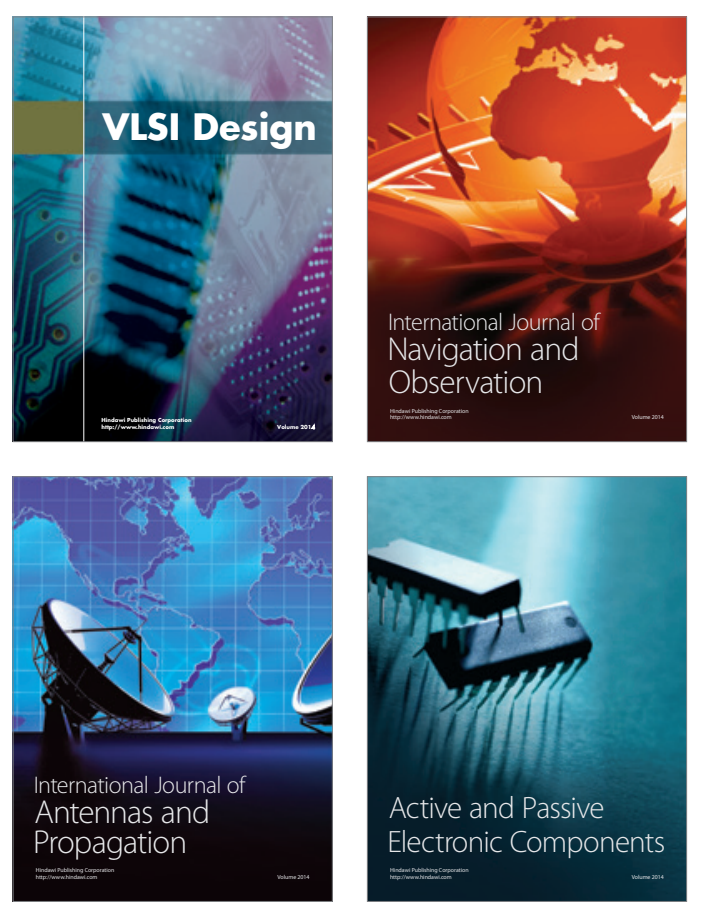
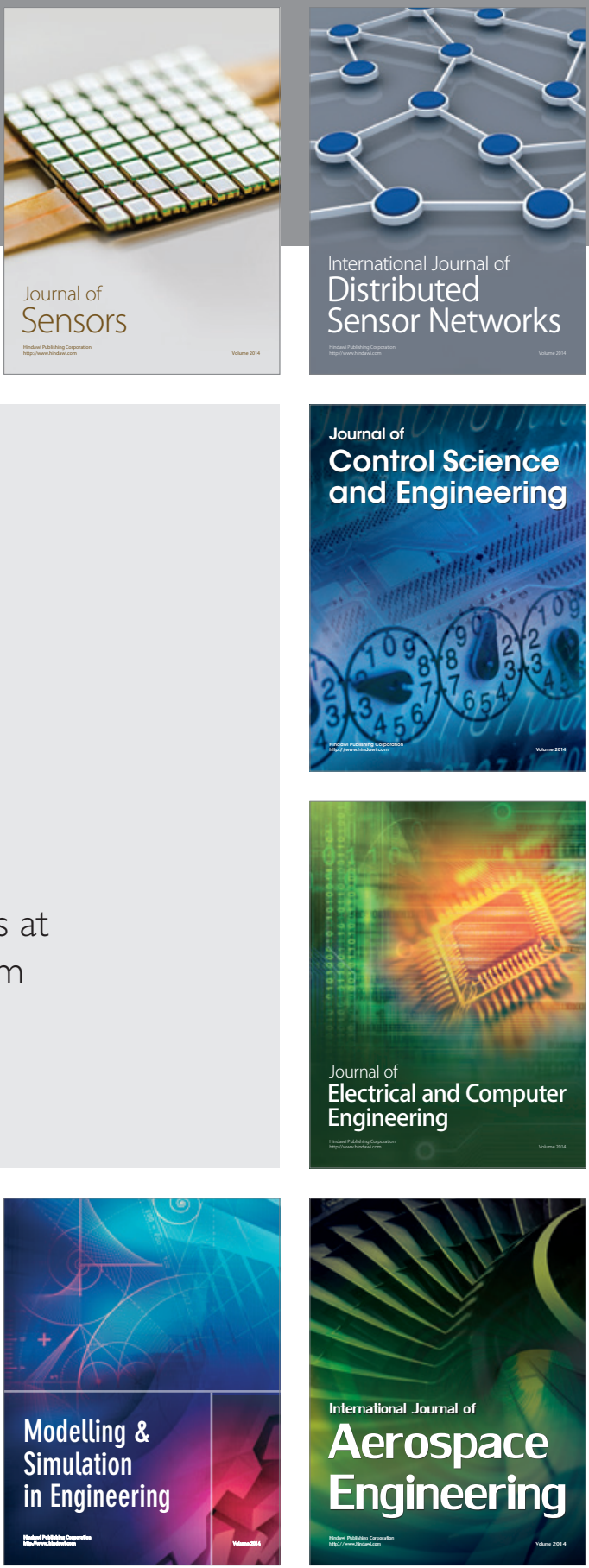

Journal of

Control Science

and Engineering
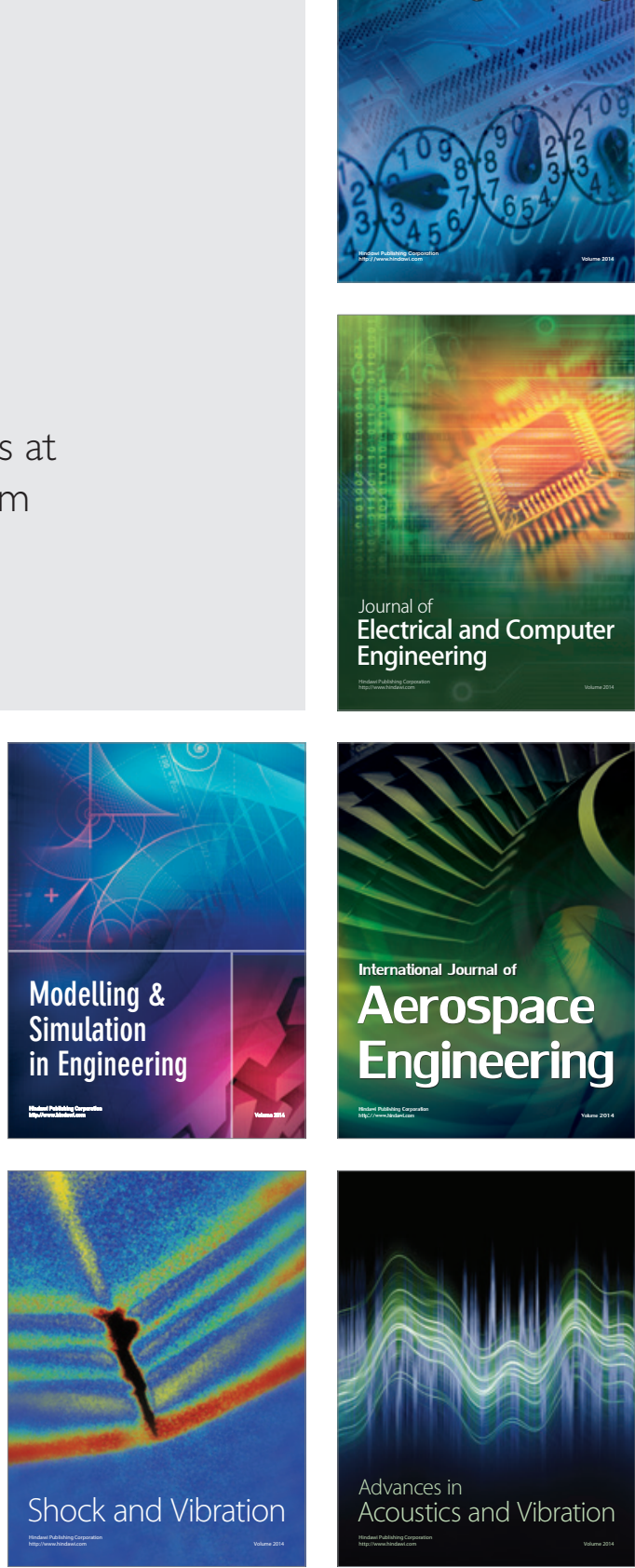